Research Paper

\title{
A Prognostic Score for Nasopharyngeal Carcinoma with Bone Metastasis: Development and Validation from Multicenter
}

\author{
Chen Chen ${ }^{1,2}$, Jing-Bo Wu ${ }^{3}$, Hao Jiang,4, Jin Gao5, Jia-Xin Chen ${ }^{6}$, Chang-Chuan Pan7, Lu-Jun Shen ${ }^{2,8}, \mathrm{Yu}$ \\ Chen ${ }^{3}$, Hui Chang ${ }^{1,2}$, Ya-Lan Tao ${ }^{1,2}$, Xiao-Hui Li ${ }^{1,2}$, Pei-Hong Wu ${ }^{2,8}$, Yun-Fei Xia ${ }^{1,2}$ \\ 1. Department of Radiation Oncology, Sun Yat-sen University Cancer Center, Guangzhou, People's Republic of China \\ 2. State Key Laboratory of Oncology in South China; Collaborative Innovation Center for Cancer Medicine, Guangzhou, People's Republic of China \\ 3. Department of Oncology, The affiliated hospital of Luzhou medical college, Luzhou, People's Republic of China \\ 4. Department of Radiation Oncology, The first affiliated hospital of Bengbu medical college, Bengbu, People's Republic of China \\ 5. Department of Radiation Oncology, Anhui Provincial Hospital, Hefei, People's Republic of China \\ 6. Department of Radiation Oncology, The people's hospital of Guangxi zhuang autonomous region, Nanning, People's Republic of China \\ 7. Department of Medical Oncology, Sichuan Cancer Hospital and Institute, Chengdu, People's Republic of China \\ 8. Departments of Medical Imaging and Interventional Radiology, Sun Yat-sen University Cancer Center, Guangzhou, People's Republic of China \\ $\square$ Corresponding author: Yun-Fei Xia, 651 Dongfeng Road East, Guangzhou 510060, People's Republic of China. Tel: 86-20-87343169; Fax:86-20-87343294; \\ E-mail: xiayf@sysucc.org.cn \\ (c) Ivyspring International Publisher. This is an open access article distributed under the terms of the Creative Commons Attribution (CC BY-NC) license \\ (https://creativecommons.org/licenses/by-nc/4.0/). See http://ivyspring.com/terms for full terms and conditions.
}

Received: 2017.09.03; Accepted: 2017.12.04; Published: 2018.02.12

\begin{abstract}
Background: To establish a prognostic score based on clinical routine factors to stratify nasopharyngeal carcinoma patients with bone metastasis into risk groups with different survival rates.

Materials and Methods: Total 276 patients from multicenter were retrospectively analyzed. Kaplan-Meier method and Cox regression were used to confirm independent risk factors, which were checked for internal validity by bootstrapping method. The prognostic score, deriving from the corresponding regression coefficients in Cox model, classified patients into low and high risk groups. Finally, two independent cohorts were used for external validation.

Results: In development cohort, six risk factors were identified: age $>46$ year-old (point $=1$ ), $N>$ 0 stage (point=2), anemia (point $=2$ ), bone metastasis free interval $\leq 12$ months (point $=1$ ), without radiotherapy to primary sites (point $=1$ ), and without radiotherapy to first metastasis sites (point $=1$ ). The derived prognostic score divided patients into low (score, 0-4) and high (score, 5-8) risk groups, with highly significant differences of 5 -year overall survival rates (high vs. low risk: $24.6 \%$ vs. $58.2 \%$, HR 3.47, $P<0.001)$. Two external validations presented congruent results.

Conclusion: A feasible and applicative prognostic score was successfully established and validated to discriminate bone metastatic nasopharyngeal carcinoma into low/high risk groups, which will be useful for individual treatment.
\end{abstract}

Key words: Prognostic score; nasopharyngeal carcinoma; bone metastasis; validation; multicenter.

\section{Introduction}

Nasopharyngeal carcinoma (NPC) remains high incidence rate in endemic regions such as Southern China[1]. In contrast to other squamous cell carcinoma of the head and neck, NPC is characterized by a high tendency for metastasis. The distant metastases rate ranges from $11 \%$ to $36 \%[2,3]$. With the improvement in local control with the application of high-precision radiotherapy, distant metastasis failure is expected to become an increasingly predominant cause of death from NPC[4]. 
NPC patients with metastasis make a very heterogeneous group with the overall survival ranging from weeks to years[5]. Interestingly, there has been reported that the patient with solitary bone metastasis (BM) can obtain long term survival by active treatment[6]. Thus, we postulate that it may be associated with a unique biologic behavior in NPC patients with BM. There is a need for a score system to predict survival risk of them.

Few studies have been reported focusing on prognostic score of NPC patients with BM. Xun Cao et al[7] enrolled 116 NPC patients with BM and divided them into different risk groups based on the independently significant prognostic factors including age, local recurrence, subsequent metastasis, disease-free interval and treatment modality. However, the factors they collected did not contain the laboratory routine parameters, such as platelet (PLT), hemoglobin (HGB), lactic dehydrogenase (LDH) and so on, which were associated with

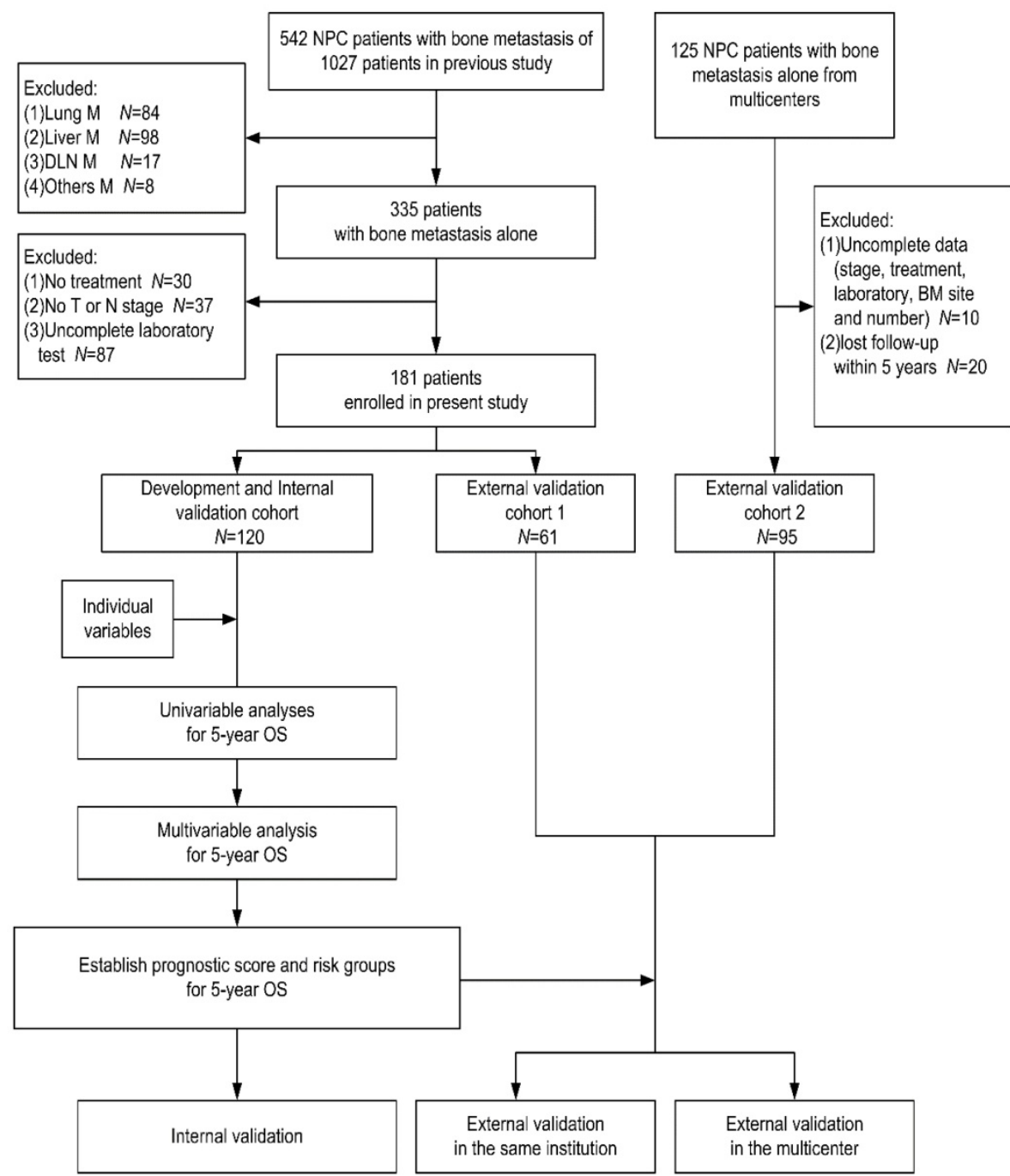

Fig. 1. The population and design of study. Individual variables were the variables we listed in the Table 1. Abbreviations: NPC: nasopharyngeal carcinoma; M: metastasis; DLN: distant lymph node; BM: bone metastasis; OS: overall survival. metastasis of NPC in our previous researches[8, 9]. Therefore, the purpose of our present study is to establish a simple and convenient prognostic score based on routine factors to stratify patients into different risk groups.

\section{Materials and Methods}

\section{Population}

In the previous study[10], we had identified 1027 patients with a confirmed clinical diagnosis of metastatic NPC in our institution between January 1995 and December 2007 continuously. In the present study, we selected 181 of 542 BM patients from mentioned-above population according to the criteria as follows: (1) only bone involved at the primary metastasis, (2) complete records of BM locus and number, (3) to receive treatment after diagnosis of NPC and BM, at least either chemotherapy or radiotherapy, (4) with a definite survival status. The patients with incomplete clinical data were excluded. Based on the criteria above, we further enrolled 95 patients from other four institutions, including the affiliated hospital of Luzhou medical college, the first affiliated hospital of Bengbu medical college, Anhui provincial hospital, and the people's hospital of Guangxi zhuang autonomous region.

We randomized the patients from our center into two cohorts according to 2:1 ratio: the one (120 patients) was development cohort for establishing the prognostic score, and the other one (61 patients) was external validation cohort 1 for our center validation of the established score. The patients from other four institutions were defined as external validation cohort 2 for independent external validation. The study population was presented in Fig 1.

\section{Pretreatment assessment and data collection}

Each patient in our study had received a pretreatment assessment at initial diagnosis and BM occurrence, including complete history-taking, phys- 
ical examination, fiberoptic nasoendoscopy, hematology and biochemistry routine test, Epstein-Barr virus (EBV) serology, chest radiographs, abdomen ultrasound examination, emission computed tomography (ECT) of bone and magnetic resonance imaging (MRI) of the nasopharynx and neck. Some of these patients had also received computed tomography (CT)/MRI/X-ray of bone and positron emission tomography-computed tomography (PET/CT). Hematological tests were applied within 2 weeks of BM occurrence.

The data we collected included: (1) gender, age, and Karnosky performance score (KPS) at the time of metastasis, (2) hematology and biochemistry routine test parameters, EBV serology VCA-IgA titre and EA-IgA titre within 2 weeks of BM occurrence, (3) UICC/AJCC stage (7th edition), pathology and the initial time of diagnosis, (4) BM locus, BM number, the time of first occurrence of BM, (5) chemotherapy and/or radiotherapy for the primary sites and first metastasis sites, (6) the definite survival status and the time of death. In our study, BM loci included ribs, sternum, cervical vertebrae, thoracic vertebrae, lumbar vertebrae, sacral vertebrae, shoulder joint, hip joint, pelvis, long bones of the upper extremity, long bones of the lower extremity, and other bones. BM number referred to the number of metastatic tumors of bone regardless of the locus.

Anatomy of BM was classified in three patterns according to locus, number, and side of BM, respectively. Classification of $\mathrm{BM}$ locus was defined as: (1) solitary locus $(n=1)$, (2) oligo-loci $(1<n \leq 3)$, (3) multi-loci $(n>3)$. Classification of BM number was defined as: (1) solitary number $(n=1)$, oligo-number $(1<n \leq 3)$, (3) multi-number $(n>3)$. Classification of BM side was defined as: (1) upper side (metastases only above/at the 12nd thoracic vertebrae), (2) lower side (metastases only below the 12nd thoracic vertebrae), (3) both side (metastases at upper and lower sides). Bone metastasis free interval (BMFI) was defined as time from initial diagnosis to the first occurrence of BM.

Laboratory parameters were categorized according to the normal range of each item. The cut-off value of EBV serology VCA-IgA titre and EA-IgA titre were referred to the study reported by Xun Cao et al[7].

\section{Treatment}

Radiotherapy (RT) for nasopharynx and neck included conventional 2-dimension (2D) RT, 3-dimension (3D) RT and intensity-modulated radiotherapy (IMRT). All three kinds of RT were conducted according to the RT guideline of our cancer, which has been described in our previously study[11]. Bone metastatic tumors were irradiated in the radiation treatment schedules including $40 \mathrm{~Gy} / 20$ fraction, 30Gy/10 fraction, 20Gy/5 fraction, 20Gy/4 faction, and $8 \mathrm{~Gy} / 1$ fraction. Chemotherapy was platinum-based combination chemotherapy or concurrent chemo/RT or clinical trial chemotherapy. Other therapies included zoledronic acid and surgery treatment for metastatic bone. The treatments of other four institutions were the same with ours in principle.

\section{Follow-up, endpoints and statistical analysis}

The patients were subsequently followed up every 3 months during the first 3 years, every 6 months during the next 2 years, and then annually. The last date of follow-up was August, 2014. The endpoint of our study was overall survival (OS) defined as time from diagnosis to death from any cause.

All categorical data were described using numbers and percentages, and compared by X2 test. Quantitative data were presented using median and range. Survival curves were drawn using the Kaplan-Meier method and pair-wise comparisons were performed with the two-side log-rank test. Multivariable analyses were performed by Cox regression in backward conditioned method with corresponding hazard ratio (HR), 95\% confidence interval $(\mathrm{CI})$ and probability $(\mathrm{P})$ value estimated. The association of two factors was tested by contingency table analysis. Receiver operating characteristic (ROC) curves of BM classifications were depicted and the comparison of area under the curve (AUC) was made to select the best prediction efficiency on 5-year OS. All tests were two-tailed, with P value of less than 0.05 considered statistically significant. Statistical analyses were performed by IBM Statistical Product and Service Solutions (IBM SPSS) statistics, version 21.0.

\section{Study design}

The flowchart of our study design was presented in Fig 1. Firstly, in the development cohort, we evaluated the impact of each variable on survival to identify the potential risk factor of prognosis $(\mathrm{P}<0.1$ in univariate analysis), which were accepted into the multivariable analysis. For the three patterns of BM classification to be likely the potential risk factors, we only chose one with best efficiency of survival prediction into the multivariable analyses. For variables with more than two subsets, pair-wise comparisons were used to combine undifferentiated subsets. In multivariate analysis, the factors that maintained in the final regression equation were considered as independent prognostic factors. Bootstrapping method, which resampled to $n=1000$, was used to check the internal validity and stability of the Cox regression equation. Then, each factor was 
assigned a definite point based on the linear transformation of the corresponding $\beta$ regression coefficient divided by the lowest $\beta$ value and rounded to the nearest integer. The prognostic score was the sum of points. Furthermore, the derived prognostic score discriminated two, which have low and high, risk groups by merging patients with various prognostic score according to undifferentiated 5-year overall survival rates. Finally, we validated the prognostic score in external cohort 1 and 2 . This study was approved by the Institutional Review Board of Sun Yat-Sen University Cancer Center.

\section{Results}

\section{Demographics}

The baseline characteristics of the three cohorts were listed in Table 1. Ether in development or validation cohorts, the main types of pathology were poorly differentiated squamous cell carcinoma and undifferentiated non-keratinizing carcinoma, which accounted for more than 90 percent of all. All BM were diagnosed by combination of at least two imaging examinations. The lost of follow-up rate within 5 years were $18.3 \%, 13.1 \%$, and $3.2 \%$ for above cohorts, respectively.

\section{Survival analyses}

Until the end of follow-up, there were 83 patients of development cohort, 40 patients of external validation cohort 1 , and 73 patients of external validation cohort 2 died (D). The median OS time of above cohorts was 36.9 months (95\% CI, 29.7-44.2), 33.4 months (95\% CI, 15.5-51.2) and 29.6 months $(95 \%$ CI, 23.3-35.8), respectively.

OS rates of 1,3 , and 5 years were $87.5 \%(D=15)$, $55.8 \%(D=53)$ and $40.0 \%(D=72)$ for development cohort, $91.8 \%(D=5), 50.8 \%(D=30)$ and $45.9 \%(D=33)$ for external validation cohort 1 , and $78.9 \%(D=20)$, $37.9 \%(D=59)$ and $23.2 \%(D=73)$ for external validation cohort 2.

\section{Establishment of prognostic score}

Univariate analyses of 5-year OS in development cohort were listed in Table 1. BM classifications by locus, number and side were all potential risk factors of 5-year OS, and BM side classification had the largest AUC of ROC curve (AUC $=0.588,0.604$, and 0.632 for BM locus, number and side classifications, respectively). Further pair-wise comparisons of subsets for BM side classification showed that 5-year OS rate of upper and lower side BM had no significant difference $(\mathrm{P}=0.966)$, while they were both significantly higher than that of both side BM ( $P=0.019$ for upper and 0.041 for lower side). Thus, we combined upper and lower side as the new subset named single side, which still had significantly higher 5 -year OS rate $(57.8 \%)$ than that of both side BM (29.3\%) ( $\mathrm{P}=0.004)$. Similarly, we merged N1 to N3 stage (all $\mathrm{P}>0.1$ in pair-wise comparisons) into non-N0 stage, with significantly lower 5-year OS rate $(36.9 \%)$ than that of N0 stage $(58.8 \%) \quad(P=0.015)$. Finally we selected the new binary BM side and $\mathrm{N}$ stage classification, together with other potential risk factors, into multivariate analysis. In addition, 21 patients received multi-cycle $(>6)$ chemotherapy after BM and 84 patients received short cycle $(\leq 6)$, with no significant difference of 5 -year OS $(33.3 \%$ vs. $44.0 \%, \mathrm{P}=0.676$ ).

In the multivariate analyses, six risk factors were finally determined for 5-year OS, including age $>46$ year-old, $\mathrm{N}>0$ stage, anemia, BMFI $\leq 12$ months, without RT for primary and first metastatic sites (Table 2).

Bootstrapping was then performed and resulted in a very high consistency and stability of the risk factors we obtained above (Table 2).

The points for each risk factor were presented in Table 2. The prognostic score (range, 0-8) was calculated for each patient in development cohort and discriminated two risk groups, which had low (score 0-4) and high (score 5-8) risks, with highly significant differences of 5-year OS rates (high compared with low risk: HR 3.47, $\mathrm{P}<0.001$ ) (Figure 2A).

\section{Validation}

Prognostic score calculation and risk group division were repeated in two external validation cohorts. The distributions of risk factors in two validation cohorts were mostly consist with that in development cohort (Table 3). Survival curves of 5 -year OS by risk groups also showed significant differences in both external validation cohort 1 (high compared with low risk: HR 2.70, $\mathrm{P}=0.004$, Figure 2B) and cohort 2 (high compared with low risk: HR 1.90, $\mathrm{P}=0.007$, Figure $2 \mathrm{C}$ ), which were similar to the results of development cohort regardless of the origin of patients.

\section{Discussion}

We established the prognostic score for NPC with $\mathrm{BM}$ involving six risk factors, including age $>46$ year-old (point=1), $\mathrm{N}>0$ stage (point=2), anemia (point=2), BMFI $\leq 12$ months (point=1), without RT to primary sites (point $=1$ ), and without RT to first BM sites (point $=1$ ), and stratified patients into low (score, 0-4) and high (score, 5-8) risk groups with significantly different 5-year OS rates either in development cohort or in external validation cohorts. 
Table 1. The baseline characteristics of patients in three cohorts and univariate analyses of 5-year overall survival in development cohort

\begin{tabular}{|c|c|c|c|c|c|c|c|}
\hline \multirow[t]{2}{*}{ Characters } & \multicolumn{3}{|c|}{ Development cohort } & \multicolumn{2}{|c|}{ External validation cohort 1} & \multicolumn{2}{|c|}{ External validation cohort 2} \\
\hline & $\mathbf{N}(\%)$ & $\mathrm{D}_{5-\mathrm{y}}$ os $(\mathbf{R} \%)$ & Uni-P5-y os & N (\%) & $\mathbf{P}_{1}$ & N (\%) & $\mathbf{P}_{2}$ \\
\hline \multicolumn{8}{|l|}{ Age (year-old) } \\
\hline Median (range) & $46(11-73)$ & & & $47(21-66)$ & & $51(16-72)$ & \\
\hline$\leq 46$ & $67(55.8)$ & $34(50.7)$ & 0.017 & $28(45.9)$ & 0.440 & $35(36.8)$ & 0.006 \\
\hline$>46$ & $53(44.2)$ & $38(71.7)$ & & $33(54.1)$ & & $60(63.2)$ & \\
\hline \multicolumn{8}{|l|}{ Gender } \\
\hline Male & 104(86.7) & $64(61.5)$ & 0.366 & $51(83.6)$ & 0.929 & 75 (78.9) & 0.132 \\
\hline Female & $16(13.3)$ & $8(50.0)$ & & $10(16.4)$ & & $20(21.1)$ & \\
\hline \multicolumn{8}{|l|}{ KPS } \\
\hline$\leq 80$ & 69 (57.5) & $42(60.9)$ & 0.493 & $30(49.2)$ & 0.869 & $41(43.2)$ & 0.775 \\
\hline$>80$ & $51(42.5)$ & $30(58.8)$ & & $31(50.8)$ & & $33(34.7)$ & \\
\hline unknown & NA & NA & & NA & & $21(22.1)$ & \\
\hline \multicolumn{8}{|l|}{ UICC T stage } \\
\hline $\mathrm{T} 1$ & $22(18.3)$ & $14(63.6)$ & 0.238 & $10(16.4)$ & 0.507 & $4(4.2)$ & $<0.001$ \\
\hline $\mathrm{T} 2$ & $31(25.8)$ & $15(48.4)$ & & $13(21.3)$ & & $23(24.2)$ & \\
\hline T3 & $56(46.7)$ & $36(64.3)$ & & $19(31.1)$ & & $40(42.1)$ & \\
\hline $\mathrm{T} 4$ & $11(9.2)$ & $7(63.6)$ & & 19 (31.2) & & $28(29.5)$ & \\
\hline \multicolumn{8}{|l|}{ UICC N stage } \\
\hline No & $17(14.2)$ & $7(41.2)$ & 0.085 & $10(16.4)$ & 0.094 & $5(5.3)$ & 0.090 \\
\hline N1 & $41(34.2)$ & $24(58.5)$ & & $20(32.8)$ & & $28(29.5)$ & \\
\hline N2 & $37(30.8)$ & $25(67.6)$ & & $19(31.1)$ & & $40(42.1)$ & \\
\hline N3 & $25(20.8)$ & $16(64.0)$ & & $12(19.7)$ & & $22(23.1)$ & \\
\hline \multicolumn{8}{|l|}{ WBC $\left(10^{9} / \mathrm{L}\right)$} \\
\hline$\leq 10$ & 103(85.8) & $60(58.3)$ & 0.011 & $57(93.4)$ & 0.628 & $86(90.5)$ & 0.295 \\
\hline$>10$ & $17(14.2)$ & $12(70.6)$ & & $4(6.6)$ & & $9(9.5)$ & \\
\hline \multicolumn{8}{|l|}{ HGB $(\mathrm{g} / \mathrm{L})$} \\
\hline$\leq 130^{*} / 120 \Delta$ & 49 (40.8) & $38(77.6)$ & $<0.001$ & 15 (24.6) & 0.967 & 49 (51.6) & 0.116 \\
\hline$>130^{*} / 120 \Delta$ & $71(59.2)$ & $34(47.9)$ & & $46(75.4)$ & & $46(48.4)$ & \\
\hline \multicolumn{8}{|l|}{ PLT (109/L) } \\
\hline$\leq 300$ & 88 (73.3) & $50(56.8)$ & 0.225 & 48 (78.7) & 0.307 & $80(84.2)$ & 0.055 \\
\hline$>300$ & $32(26.7)$ & $22(68.8)$ & & $13(21.3)$ & & 15 (15.8) & \\
\hline LDH (U/L) & & & & & & & \\
\hline$\leq 245$ & $80(66.7)$ & $45(56.3)$ & 0.015 & $41(67.2)$ & 0.415 & $27(28.4)$ & 0.205 \\
\hline$>245$ & $40(33.3)$ & $27(67.5)$ & & $20(32.8)$ & & $21(22.1)$ & \\
\hline unknown & NA & NA & & $\mathrm{NA}$ & & 47 (49.5) & \\
\hline $\operatorname{ALP}(\mathrm{U} / \mathrm{L})$ & & & & & & & \\
\hline$\leq 110$ & 87 (72.5) & $51(58.6)$ & 0.548 & $42(68.9)$ & 0.325 & $56(58.9)$ & 0.856 \\
\hline$>110$ & $33(27.5)$ & $21(63.6)$ & & $12(19.7)$ & & $20(21.1)$ & \\
\hline unknown & NA & NA & & NA & & $19(20.0)$ & \\
\hline EBV VCA-IgA $t$ & & & & & & & \\
\hline$\leq 1: 320$ & $56(46.7)$ & $31(55.4)$ & 0.253 & $29(47.6)$ & 0.421 & NA & NA \\
\hline$>1: 320$ & $24(20.0)$ & $17(70.8)$ & & $16(26.2)$ & & NA & \\
\hline Unknown & $40(33.3)$ & $24(60.0)$ & & $16(26.2)$ & & $95(100)$ & \\
\hline EBV EA-IgA tit & & & & & & & \\
\hline$\leq 1: 40$ & $57(47.5)$ & $32(56.1)$ & 0.602 & $31(50.8)$ & 0.202 & NA & NA \\
\hline$>1: 40$ & $23(19.2)$ & $16(69.6)$ & & $14(23.0)$ & & NA & \\
\hline Unknown & 40 (33.3) & $24(60.0)$ & & $16(26.2)$ & & $95(100)$ & \\
\hline BMFI (month) & & & & & & & \\
\hline$>12$ & $33(27.5)$ & $17(51.5)$ & 0.005 & $16(26.2)$ & 0.753 & $31(32.6)$ & 0.414 \\
\hline$\leq 12$ & $87(72.5)$ & $55(63.2)$ & & $45(73.8)$ & & $64(67.4)$ & \\
\hline BM locus & & & & & & & \\
\hline Solitary & $30(25.0)$ & $14(46.7)$ & 0.045 & $15(24.6)$ & 0.253 & 47 (49.5) & $<0.001$ \\
\hline Oligo-loci & $41(34.2)$ & $25(61.0)$ & & $22(36.1)$ & & 37 (38.9) & \\
\hline Multi-loci & $49(40.8)$ & $33(67.3)$ & & $24(39.3)$ & & 11 (11.6) & \\
\hline BM number & & & & & & & \\
\hline Solitary & $22(18.4)$ & $9(40.9)$ & 0.072 & $10(16.4)$ & 0.019 & $30(31.6)$ & $<0.001$ \\
\hline Oligo-numb & 19 (15.8) & $10(52.6)$ & & $12(19.7)$ & & $41(43.1)$ & \\
\hline Multi-numb & 79 (65.8) & $53(67.1)$ & & $39(63.9)$ & & $24(25.3)$ & \\
\hline BM side & & & & & & & \\
\hline Upper side & $26(21.7)$ & $12(46.2)$ & 0.017 & $13(21.3)$ & 0.924 & $39(41.1)$ & 0.002 \\
\hline Lower side & $19(15.8)$ & $7(36.8)$ & & $8(13.1)$ & & 18 (18.9) & \\
\hline Both sides & $75(62.5)$ & $53(70.7)$ & & $40(65.6)$ & & $38(40.0)$ & \\
\hline Subsequent me & & & & & & & \\
\hline Yes & $28(23.3)$ & $16(57.1)$ & 0.479 & $11(18.0)$ & 0.881 & $31(32.6)$ & 0.129 \\
\hline No & $92(76.7)$ & $56(60.9)$ & & $50(82.0)$ & & $64(67.4)$ & \\
\hline
\end{tabular}




\begin{tabular}{|c|c|c|c|c|c|c|c|}
\hline \multirow[t]{2}{*}{ Characters } & \multicolumn{3}{|c|}{ Development cohort } & \multicolumn{2}{|c|}{ External validation cohort 1} & \multicolumn{2}{|c|}{ External validation cohort 2} \\
\hline & $\mathbf{N}(\%)$ & $\mathrm{D}_{5-\mathrm{y}}$ os $(\mathrm{R} \%)$ & Uni-P $_{5-\mathrm{y}}$ os & $\mathbf{N}(\%)$ & $\mathbf{P}_{1}$ & $\mathbf{N}(\%)$ & $\mathbf{P}_{2}$ \\
\hline Yes & $96(80.0)$ & $55(57.3)$ & $<0.001$ & $46(75.4)$ & 0.354 & $87(91.6)$ & 0.018 \\
\hline No & $24(20.0)$ & $17(70.8)$ & & $15(24.6)$ & & $8(8.4)$ & \\
\hline \multicolumn{8}{|c|}{ First metastasis site RT } \\
\hline Yes & $44(36.7)$ & $20(45.5)$ & $<0.001$ & $19(31.1)$ & 0.224 & $52(54.7)$ & 0.008 \\
\hline No & $76(63.3)$ & $52(68.4)$ & & $42(68.9)$ & & $43(45.3)$ & \\
\hline \multicolumn{8}{|c|}{ Progress after first metastasis } \\
\hline Yes & $57(47.5)$ & $36(63.2)$ & 0.944 & $29(47.5)$ & 0.669 & $44(46.3)$ & 0.863 \\
\hline No & $63(52.5)$ & $36(57.1)$ & & $32(52.5)$ & & $51(53.7)$ & \\
\hline
\end{tabular}

Table 2. Multivariate analysis of 5-year overall survival and internal validation by bootstrapping in development cohort

\begin{tabular}{|c|c|c|c|c|c|c|c|c|c|c|c|c|c|c|c|}
\hline \multirow{3}{*}{$\begin{array}{l}\text { Variables in the } \\
\text { equation }\end{array}$} & \multicolumn{9}{|c|}{ Multivariate analysis by Cox regression } & \multicolumn{6}{|c|}{ Internal validation by bootstrap method } \\
\hline & \multirow[t]{2}{*}{$\bar{B}$} & \multirow[t]{2}{*}{ SE } & \multirow[t]{2}{*}{ Wald } & \multirow[t]{2}{*}{ df } & \multirow[t]{2}{*}{ Significance } & \multirow[t]{2}{*}{$\operatorname{Exp}(B)$} & \multicolumn{2}{|c|}{$\begin{array}{l}95 \% \text { CI for Exp } \\
\text { (B) }\end{array}$} & \multirow[t]{2}{*}{ Point } & \multirow[t]{2}{*}{$\overline{\mathbf{B}}$} & \multirow[t]{2}{*}{ Bias } & \multirow[t]{2}{*}{$\begin{array}{l}\text { Standard } \\
\text { error }\end{array}$} & \multirow[t]{2}{*}{$\begin{array}{l}\text { Significance } \\
\text { (2-tailed) }\end{array}$} & \multicolumn{2}{|l|}{$95 \% \mathrm{CI}$} \\
\hline & & & & & & & Lower & Upper & & & & & & Lower & Upper \\
\hline \multicolumn{16}{|l|}{ Age (year-old) } \\
\hline$\leq 46$ vs. $>46$ & 0.655 & 0.259 & 6.391 & 1 & 0.011 & 1.926 & 1.159 & 3.200 & 0,1 & 0.638 & 0.007 & 0.325 & 0.037 & 0.025 & 1.332 \\
\hline \multicolumn{16}{|l|}{ UICC N stage } \\
\hline N0 vs. $N>0$ & 1.120 & 0.426 & 6.926 & 1 & 0.008 & 3.065 & 1.331 & 7.058 & 0,2 & 1.106 & 0.082 & 0.632 & 0.046 & 0.007 & 2.575 \\
\hline \multicolumn{16}{|l|}{ HGB (g/L) } \\
\hline $\begin{array}{l}>130^{*} / 120^{\star} \text { Vs. } \\
\leq 130^{*} / 120^{\star}\end{array}$ & 1.429 & 0.273 & 27.336 & 1 & $<0.001$ & 4.174 & 2.443 & 7.132 & 0,2 & 1.479 & 0.105 & 0.378 & 0.001 & 0.912 & 2.394 \\
\hline \multicolumn{16}{|l|}{ BMFI (month) } \\
\hline$>12$ vs. $\leq 12$ & 0.943 & 0.331 & 8.137 & 1 & 0.004 & 2.569 & 1.343 & 4.912 & 0,1 & 0.984 & 0.086 & 0.401 & 0.009 & 0.332 & 1.980 \\
\hline \multicolumn{16}{|l|}{ Primary site RT } \\
\hline Yes vs. No & 0.929 & 0.323 & 8.292 & 1 & 0.004 & 2.532 & 1.345 & 4.766 & 0,1 & 0.934 & 0.075 & 0.446 & 0.019 & 0.193 & 1.895 \\
\hline \multicolumn{16}{|c|}{ First metastasis site RT } \\
\hline Yes vs. No & 0.746 & 0.316 & 5.561 & 1 & 0.018 & 2.108 & 1.134 & 3.919 & 0,1 & 0.850 & 0.040 & 0.398 & 0.024 & 0.169 & 1.665 \\
\hline
\end{tabular}

Footnote: *for male; $\mathbf{\Delta}$ for female.

Abbreviations: UICC: International Union Against Cancer; BMFI: bone metastasis free interval; RT: radiotherapy.

Table 3. Risk factors distributions of three cohorts in low and high risk groups

\begin{tabular}{|c|c|c|c|c|c|c|c|c|}
\hline \multirow[t]{3}{*}{ Factors } & \multicolumn{4}{|c|}{ Low risk group (score, $0-4$ ) } & \multicolumn{4}{|c|}{ High risk group (score, $5-8$ ) } \\
\hline & $\begin{array}{l}\text { Development } \\
\text { cohort }(N=55)\end{array}$ & $\begin{array}{l}\text { External validation } \\
\text { cohort } 1(\mathrm{~N}=31)\end{array}$ & $\begin{array}{l}\text { External validation } \\
\text { cohort } 2(\mathrm{~N}=42)\end{array}$ & $\mathbf{P}_{1}$ & $\begin{array}{l}\text { Development } \\
\text { cohort }(\mathrm{N}=65)\end{array}$ & $\begin{array}{l}\text { External validation } \\
\text { cohort } 1(\mathrm{~N}=30)\end{array}$ & $\begin{array}{l}\text { External validation } \\
\text { cohort } 2(\mathrm{~N}=53)\end{array}$ & $\mathbf{P}_{2}$ \\
\hline & $\mathbf{N}(\%)$ & $\mathbf{N}(\%)$ & N (\%) & & N (\%) & $\mathbf{N}(\%)$ & $\mathbf{N}(\%)$ & \\
\hline \multicolumn{9}{|c|}{ Age (point, 0 vs. 1 ) } \\
\hline$\leq 46$ & $41(74.5)$ & $21(67.7)$ & $21(50.0)$ & 0.040 & $26(40.0)$ & $7(23.3)$ & $14(26.4)$ & 0.156 \\
\hline$>46$ & $14(25.5)$ & $10(32.3)$ & $21(50.0)$ & & $39(60.0)$ & $23(76.7)$ & $39(73.6)$ & \\
\hline \multicolumn{9}{|c|}{ UICC N stage (point, 0 vs. 2 ) } \\
\hline No & $16(29.1)$ & $9(29.0)$ & $5(11.9)$ & 0.099 & $1(1.5)$ & $1(3.3)$ & $0(0.0)$ & 0.443 \\
\hline$N>0$ & $39(70.9)$ & $22(71.0)$ & $37(88.1)$ & & $64(98.5)$ & $29(96.7)$ & $53(100.0)$ & \\
\hline \multicolumn{9}{|c|}{ HGB (g/L) (point, 0 vs. 2) } \\
\hline$>130^{*} / 120 \Delta$ & $46(83.6)$ & $30(96.8)$ & $36(85.7)$ & 0.191 & $25(38.5)$ & $16(53.3)$ & $10(18.9)$ & 0.004 \\
\hline$\leq 130^{*} / 120 \wedge$ & $9(16.4)$ & $1(3.2)$ & $6(14.3)$ & & $40(61.5)$ & $14(46.7)$ & $43(81.1)$ & \\
\hline \multicolumn{9}{|c|}{ BMFI (month) (point, 0 vs. 1) } \\
\hline$>12$ & $23(41.8)$ & $13(41.9)$ & $22(52.4)$ & 0.532 & $10(15.4)$ & $3(10.0)$ & $9(17.0)$ & 0.683 \\
\hline$\leq 12$ & $32(58.2)$ & $18(58.1)$ & $20(47.6)$ & & $55(84.6)$ & $27(90.0)$ & $44(83.0)$ & \\
\hline \multicolumn{9}{|c|}{ Primary site RT (point, 0 vs. 1 ) } \\
\hline Yes & $52(94.5)$ & $31(100.0)$ & $41(97.6)$ & 0.357 & $44(67.7)$ & $15(50.0)$ & $46(86.8)$ & 0.001 \\
\hline No & $3(5.5)$ & $0(0.0)$ & $1(2.4)$ & & $21(32.3)$ & $15(50.0)$ & $7(13.2)$ & \\
\hline \multicolumn{9}{|c|}{ First metastasis site RT (point, 0 vs. 1) } \\
\hline Yes & $33(60.0)$ & $18(58.1)$ & $30(71.4)$ & 0.403 & $11(16.9)$ & $1(3.3)$ & $22(41.5)$ & $<0.001$ \\
\hline No & $22(40.0)$ & 13 (41.9) & $12(28.6)$ & & $54(83.1)$ & $29(96.7)$ & $31(58.5)$ & \\
\hline
\end{tabular}

Footnote: ${ }^{*}$ for male; $\mathbf{\Delta}$ for female. $\mathrm{P}_{1}$ is the $\mathrm{P}$ value of $\mathrm{X}^{2}$ test for the comparison of factors distributions among three cohort in low risk group; $\mathrm{P}_{2}$ is the $\mathrm{P}$ value of $\mathrm{X}^{2}$ test for the comparison of factors distributions among three cohort in high risk group.

Abbreviations: BMFI: bone metastasis free interval; RT: radiotherapy.

There were no associations between each other of above six factors except among BMFI, primary sites RT and first metastatic sites RT (Table 4). More patients with BMFI 12 months (64 patients) or without RT for primary sites (23 patients) did not receive $\mathrm{RT}$ for first $\mathrm{BM}$ sites. It may reflect a rapid course of disease, but there were no challenge to the principles of treatment decisions in clinic. 

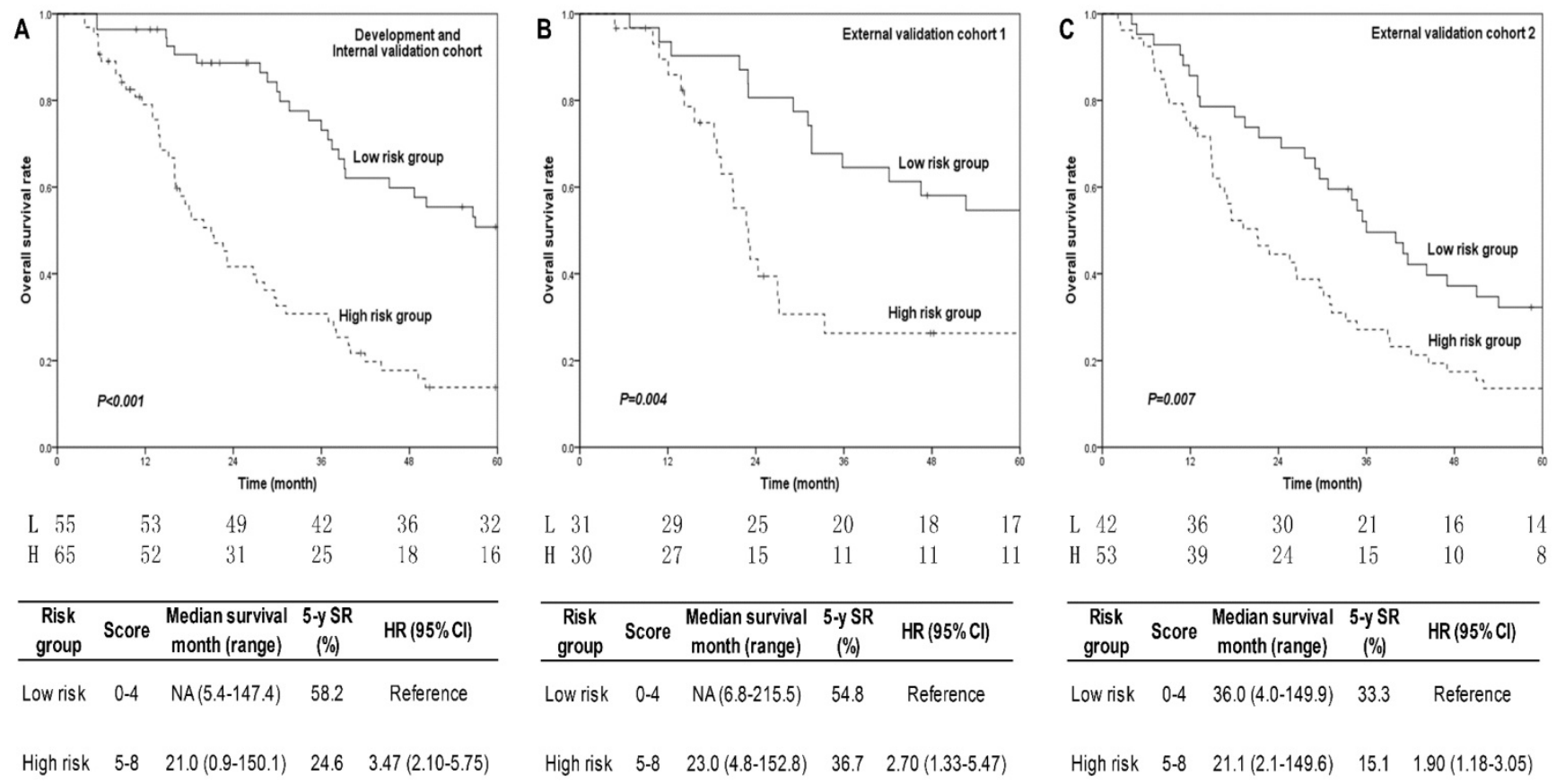

Fig. 2. 5-year overall survival curves of different risk groups in three cohorts. Fig. $2 \mathrm{~A}$ for development cohort, Fig. $2 \mathrm{~B}$ for external validation cohort 1 , and Fig. $2 \mathrm{C}$ for external validation cohort 2. P values were shown in each figure. The annual number of patients in low and high risk groups was listed under the figures. Patients in high risk group had shorter median survival time, lower 5-year survival rates, and higher hazard ratios than that in low risk group in all three cohorts. The corresponding results were present in the tables below each figure. $\mathrm{P}<0.05$ was considered statistical significance. Abbreviations: L: low risk group; H: high risk group; SR: survival rate; HR: hazard ratio.

Importantly, the biomarkers of overall functional status, such as age and HGB, which could influence the decisions of treatment, did not show any interconnections. Thus, we believe that it is necessary and scientific to perform a formal pretreatment comprehensive assessment of functional status, tumor characters, and treatment decisions.

The factors incorporated into the prognostic score were all well known and made biologic sense. Age was a common influence factor for the outcomes of many metastatic cancers, such as breast cancer[12] and colon cancer[13]. So was for NPC with BM in other's previous study[7]. It might be associated with poor tolerance of treatment.

Table 4. Interconnections between each other of six prognostic factors

\begin{tabular}{|c|c|c|c|c|c|c|}
\hline $\begin{array}{ll} & \mathrm{X}^{2} \\
& \text { value } \\
\text { P value } & \end{array}$ & Age & $\begin{array}{l}\text { UICC N } \\
\text { stage }\end{array}$ & HGB & BMFI & $\begin{array}{l}\text { Primary } \\
\text { site RT }\end{array}$ & $\begin{array}{l}\text { First } \\
\text { metastasis } \\
\text { site RT }\end{array}$ \\
\hline Age & & 3.388 & 0.058 & 3.548 & 4.089 & 2.860 \\
\hline UICC $N$ stage & 0.112 & & 1.069 & 0.603 & 0.840 & 2.259 \\
\hline HGB & 0.853 & 0.426 & & 1.103 & 1.043 & 0.614 \\
\hline BMFI & 0.067 & 0.558 & 0.307 & & 5.528 & 14.257 \\
\hline Primary site RT & 0.065 & 0.519 & 0.357 & 0.021 & & 13.645 \\
\hline $\begin{array}{l}\text { First metastasis } \\
\text { site RT }\end{array}$ & 0.127 & 0.175 & 0.448 & $<0.001$ & $<0.001$ & \\
\hline
\end{tabular}

$\mathrm{N}$ stage, as a powerful risk factor possessing 2 points in the score, was also a risk factor to develop distant metastasis[14]. Thus, it is reasonable for us to assume that patients with positive/advanced $\mathrm{N}$ stage were more likely to progress and to develop sequent metastasis after the first metastasis and then led to poor prognosis. It was also supported by our present study that more patients with $\mathrm{N}>0$ stage progressed $(49.5 \%$ vs. $35.3 \%)$ and had sequent metastasis $(25.2 \%$ vs. $11.8 \%$ ), though without any significant differences.

Anemia was another powerful risk factor. In our previous study, we had showed that anemia pre-treatment was associated with poor survival of non-metastasis NPC[11]. For metastatic NPC, we believed that anemia was still a potential risk factor of prognosis, which was confirmed in our present study. Several theories have been well known to explain the relationship between anemia and prognosis of cancer. Anemia is a guard signal or consequence of chronic inflammation status and malnutrition, which lead to high toxicity, poor compliance and response to treatment, and final convert to poor prognosis[15-18]. Anemia can reduce $\mathrm{O} 2$ transport capacity of the blood and further reduce the $\mathrm{O} 2$ supply to the tumor. Tumor hypoxia leads to the signals including proliferation, angiogenesis, and apoptosis, which all play an important role in tumor cell survival. In addition, low blood flow caused by anemia and hypoxia in tumors can serve as an effective barrier against systemic chemo-agents. The reduction of oxygenation and decreased of DNA double-strand break repair induced by hypoxia enhance radio-resistance[19-23].

A short distant metastasis free interval had been 
put forward to be a risk factor of survival for many metastatic cancers[24], although the cut-off value was various from 6 months to 2 years in different studies. In our study, we divided the BMFI into five subsets firstly including $0, \leq 3, \leq 12, \leq 24$, and $>24$ months, and no significant difference existed in pair-wise comparisons of 5-year OS rate among first 3 subsets. Therefore, we chose the cut-off value of 12 months and also proved a short BMFI to be a risk factor for survival, which might be associated with a high tumor burden and an aggressive biologic behavior of tumor cells.

The favor role of RT for primary sites had been well established[5, 25], whereas RT for first metastasis sites also played an important role in our prognostic score for NPC with BM. RT for first BM sites will substantially reduce the number of tumor cells viable within the radiation field, resulting in shrinkage of tumor bulk. Removal of tumor from the bone then reverses metastatic-induced inflammation, enables osteoblastic repair and restores integrity of the damaged bone[26, 27].

Due to the limitation of TNM staging system in individual treatment for metastatic tumor, several evaluation systems, concerning both gross anatomy and function, have been established in many cancers[28-30], including metastatic NPC[3, 24]. For all sites of metastatic NPC, Ong YK et al[24] found that ECOG $<2$, anemia, metastasis at onset, DFI $<6$ months, liver metastasis and lung metastasis were significant risk factors of survival. For synchronous liver metastasis, Yun-Ming Tian et al[31] found that KPS, LDH, primary sites RT, number of chemotherapy cycles and response to chemotherapy were significant prognostic factors. For lung metastasis, Xu Cao et al[32] conducted a series studies and set up risk groups based on factors including age, $\mathrm{T}$ stage, $\mathrm{N}$ stage, site of metastases, secondary metastases, and disease-free interval. They also established risk subsets for NPC with BM[7], which we had described in introduction section, with the limitation of lacking laboratory routine parameters and without validation. The result of our present study, specifically for BM in NPC, partially consisted with that of above studies and was sufficiently validated. The details comparisons of patients, approaches and findings between others' previous studies above and our present study were listed in Table 5.

Table 5. Details comparisons between previous studies from others and our present study

\begin{tabular}{|c|c|c|c|c|c|c|c|c|c|c|c|}
\hline Author & Year of case & $\begin{array}{l}\text { Metastasis } \\
\text { type }\end{array}$ & $\begin{array}{l}\text { Patients } \\
\text { number }\end{array}$ & $\begin{array}{l}\text { Statistical } \\
\text { method }\end{array}$ & $\begin{array}{l}\text { Valida- } \\
\text { tion }\end{array}$ & Risk factors & Definition & Point & Group & Score & Survival \\
\hline \multirow{6}{*}{$\begin{array}{l}\text { Ong } \\
\text { YK[24] }\end{array}$} & \multirow[t]{6}{*}{ 1994-1999 } & \multirow[t]{6}{*}{ All sites } & \multirow[t]{6}{*}{220} & \multirow{6}{*}{$\begin{array}{l}\text { Cox } \\
\text { proportion } \\
\text { hazards } \\
\text { model }\end{array}$} & \multirow[t]{6}{*}{ No } & ECOG & $\geq 2$ & 4 & \multirow[t]{2}{*}{ Good risk } & \multirow[t]{2}{*}{$0-6$} & \multirow{2}{*}{$\begin{array}{l}\text { Median survival } \\
19.5 \text { months }\end{array}$} \\
\hline & & & & & & HGB & $<12 \mathrm{~g} / \mathrm{L}$ & 4 & & & \\
\hline & & & & & & Metastasis time & $\begin{array}{l}\text { at initial } \\
\text { diagnosis }\end{array}$ & 1 & \multirow[t]{2}{*}{$\begin{array}{l}\text { Intermediate } \\
\text { risk }\end{array}$} & \multirow[t]{2}{*}{$7-10$} & \multirow[t]{2}{*}{$\begin{array}{l}\text { Median survival } \\
10 \text { months }\end{array}$} \\
\hline & & & & & & Disease-free interval & $<6$ months & 4 & & & \\
\hline & & & & & & \multirow[t]{2}{*}{ Metastasis site } & Liver & 2 & \multirow[t]{2}{*}{ Poor risk } & \multirow[t]{2}{*}{$\geq 11$} & Median survival \\
\hline & & & & & & & Lung & 2 & & & 5.8 months \\
\hline \multirow[t]{6}{*}{ Tian[31] } & \multirow[t]{6}{*}{$2000-2009$} & \multirow[t]{6}{*}{ Liver } & \multirow[t]{6}{*}{85} & \multirow{6}{*}{$\begin{array}{l}\text { Cox } \\
\text { proportional } \\
\text { hazards } \\
\text { model }\end{array}$} & \multirow[t]{6}{*}{ No } & KPS & $\leq 70 />70$ & & & & $\begin{array}{l}\text { 3-year OS(\%) } \\
0.0 / 16.2\end{array}$ \\
\hline & & & & & & $\mathrm{LDH}$ & $\geq 245 /<$ & & & & 3-year OS(\%) \\
\hline & & & & & & & $245 \mathrm{U} / \mathrm{L}$ & & & & $9.3 / 22.6$ \\
\hline & & & & & & RT of primary tumor & No/Yes & & & & $\begin{array}{l}\text { 3-year OS(\%) } \\
5.7 / 28.1\end{array}$ \\
\hline & & & & & & Cycles of CT & $(1-5)$ vs. $\geq 6$ & & & & $\begin{array}{l}\text { 3-year OS(\%) } \\
8.6 / 18.6\end{array}$ \\
\hline & & & & & & Response to CT & No/Yes & & & & $\begin{array}{l}\text { 3-year OS(\%) } \\
0.0 / 19.2\end{array}$ \\
\hline \multirow[t]{6}{*}{ Cao[32] } & \multirow[t]{6}{*}{$1982-2000$} & \multirow[t]{6}{*}{ Lung } & \multirow[t]{6}{*}{198} & \multirow{6}{*}{$\begin{array}{l}\text { Cox } \\
\text { proportional } \\
\text { hazards } \\
\text { regression } \\
\text { model }\end{array}$} & \multirow[t]{6}{*}{ No } & Age & $>45$ year-old & 1 & \multirow[t]{2}{*}{ Low risk } & \multirow[t]{2}{*}{$0-1$} & 5 -year OS $60 \%$ \\
\hline & & & & & & T stage & T3-4 & 1 & & & \\
\hline & & & & & & $\mathrm{N}$ stage & N2-3 & 1 & Intermediate & $2-3$ & 5 -year OS 30\% \\
\hline & & & & & & Bilateral metastases & Yes & 1 & risk & & \\
\hline & & & & & & Secondary metastases & Yes & 1 & High risk & $4-8$ & 5-year OS 7\% \\
\hline & & & & & & Disease-free interval & $\leq 24$ months & 1 & & & \\
\hline Cao[7] & 1998-2000 & Bone & 116 & Cox & No & Age & $>40$ year-old & 1 & Low risk & $0-2$ & Median survival 49.5 \\
\hline & & & & proportional & & Local recurrence & Yes & 1 & & & months \\
\hline & & & & hazards & & subsequent metastasis & Yes & 1 & High risk & $3-4$ & Median survival 19.4 \\
\hline & & & & $\begin{array}{l}\text { regression } \\
\text { models }\end{array}$ & & Disease-free interval & $>24$ months & 1 & & & months \\
\hline Present & 1995-2007 & Bone & 276 & Cox & Yes & Age & $>46$ year-old & 1 & Low risk & $0-4$ & 5 -year OS $58.2 \%$ \\
\hline study & & & & proportional & & Anemia & Yes & 2 & & & \\
\hline & & & & hazards & & N stage & $\mathrm{N}>0$ & 2 & & & \\
\hline & & & & 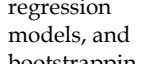 & & $\begin{array}{l}\text { Bone metastasis free } \\
\text { interval }\end{array}$ & $\leq 12$ months & 1 & High risk & $5-8$ & 5 -year OS $24.6 \%$ \\
\hline & & & & $\begin{array}{l}\text { bootstrappin } \\
\text { g method }\end{array}$ & & RT to primary sites & No & 1 & & & \\
\hline & & & & & & $\begin{array}{l}\text { RT to first metastasis } \\
\text { sites }\end{array}$ & No & 1 & & & \\
\hline
\end{tabular}


Our present study has several strengths. Firstly, we enrolled a relatively larger population of 276 patients including 95 patients from multicenter. Secondly, we conducted a long-term follow-up for survivors with the maximum survival time of more than 200 months, which can facilitate us to reveal the real differences between early deaths and long survivors. Thirdly, the risk factors we developed were checked for the validity and stability internally, and the established prognostic score was successfully validated externally in two independent cohorts. Finally and importantly, the factors in our prognostic score were all routine parameters in daily clinical practice, making the score more feasible and applicative. It is the first time to combine laboratory examination (such as blood and biochemistry routine test) with other factors, including anatomic information, patient characters and treatment approaches, to a prognostic model for NPC patients with BM.

The limitations of our present study are related to its retrospective nature. Most patients in our study are diagnosed and treated in early era. Some factors, including $\mathrm{BM}$ size, $\mathrm{C}$ reactive protein and EBV DNA load, were not assessed in our study. Two external validation cohorts' simple sizes are both relatively small, further prospective validation is needed in the future.

In conclusion, we put forward and further validated a simple and convenient prognostic score, easily measured in daily clinical practice, to evaluate the survival risk of NPC with BM at pretreatment. We believe that some bone metastatic NPC patients could obtain long survival by adaptive treatment in the modern era. Our study provides clinicians evidence to distinguish long survivors and early deaths before treatment, and further guides them to select optimal individual treatment and avoid the over or insufficient treatment.

\section{Abbreviations}

NPC: nasopharyngeal carcinoma; BM: bone metastasis; PLT: platelet; HGB: hemoglobin; LDH: lactic dehydrogenase; EBV: Epstein-Barr virus; ECT: emission computed tomography; MRI: magnetic resonance imaging; CT: computed tomography; PET/CT: positron emission tomography-computed tomography; KPS: Karnosky performance score; RT: Radiotherapy; IMRT: intensity-modulated radiotherapy; BMFI: Bone metastasis free interval; OS: overall survival; HR: hazard ratio; $\mathrm{CI}$ : confidence interval; P: probability value; ROC: receiver operating characteristic; AUC: area under the curve.

\section{Acknowledgements}

This work was supported by the Hi-Tech Research and Development Program of China (No. 2006AA02Z4B4), and National Natural Science Foundation of China (No. 31170805).

\section{Conflict of Interest}

The authors declare that they have no conflict of interest.

\section{References}

1. Wei WI, Sham JS. Nasopharyngeal carcinoma. Lancet. 2005; 365: 2041-54

2. Lee AW, Sze WM, Au JS, Leung SF, Leung TW, Chua DT, et al. Treatment results for nasopharyngeal carcinoma in the modern era: the Hong Kong experience. International journal of radiation oncology, biology, physics. 2005; 61: $1107-16$

3. Teo PM, Kwan WH, Lee WY, Leung SF, Johnson PJ. Prognosticators determining survival subsequent to distant metastasis from nasopharyngeal carcinoma. Cancer. 1996; 77: 2423-31.

4. Sun X, Su S, Chen C, Han F, Zhao C, Xiao W, et al. Long-term outcomes of intensity-modulated radiotherapy for 868 patients with nasopharyngeal carcinoma: an analysis of survival and treatment toxicities. Radiotherapy and oncology : journal of the European Society for Therapeutic Radiology and Oncology. 2014; 110: 398-403.

5. Li JX, Huang SM, Wen BX, Lu TX. Prognostic factors on overall survival of newly diagnosed metastatic nasopharyngeal carcinoma. Asian Pacific journal of cancer prevention : APJCP. 2014; 15: 3169-73.

6. Lim A, Corry J, Lau E, Rischin D. Prolonged remission in a patient with nasopharyngeal carcinoma with a solitary bone metastasis. Journal of clinical oncology : official journal of the American Society of Clinical Oncology. 2011; 29: e135-7.

7. Cao X, Han Y, He L, Xiang J, Wen Z. Risk subset of the survival for nasopharyngeal carcinoma patients with bone metastases: who will benefit from combined treatment? Oral oncology. 2011; 47: 747-52.

8. Gao J, Zhang HY, Xia YF. Increased platelet count is an indicator of metastasis in patients with nasopharyngeal carcinoma. Tumour biology : the journal of the International Society for Oncodevelopmental Biology and Medicine. 2013; 34: 39-45.

9. Li G, Gao J, Tao YL, Xu BQ, Tu ZW, Liu ZG, et al. Increased pretreatment levels of serum LDH and ALP as poor prognostic factors for nasopharyngeal carcinoma. Chinese journal of cancer. 2012; 31: 197-206.

10. Pan CC, Lu J, Yu JR, Chen P, Li W, Huang ZL, et al. Challenges in the modification of the M1 stage of the TNM staging system for nasopharyngeal carcinoma: A study of 1027 cases and review of the literature. Experimental and therapeutic medicine. 2012; 4: 334-8.

11. Gao J, Tao YL, Li G, Yi W, Xia YF. Involvement of difference in decrease of hemoglobin level in poor prognosis of Stage I and II nasopharyngeal carcinoma: implication in outcome of radiotherapy. International journal of radiation oncology, biology, physics. 2012; 82: 1471-8.

12. Largillier R, Ferrero JM, Doyen J, Barriere J, Namer M, Mari V, et al. Prognostic factors in 1,038 women with metastatic breast cancer. Annals of oncology : official journal of the European Society for Medical Oncology. 2008; 19: 2012-9.

13. Gonsalves WI, Wolpert J, Tashi T, Ganti AK, Subbiah S, Ternent C, et al. Assessment of prognostic factors after primary tumor resection in metastatic colon cancer patients: a Veteran's Affairs Central Cancer Registry (VACCR) analysis, 1995-2008. Journal of surgical oncology. 2012; 106: 486-90.

14. Xia WX, Zhang HB, Shi JL, Lu X, Wang L, Ye YF, et al. A prognostic model predicts the risk of distant metastasis and death for patients with nasopharyngeal carcinoma based on pre-treatment serum C-reactive protein and N-classification. European journal of cancer. 2013; 49: 2152-60.

15. Datema FR, Ferrier MB, Baatenburg de Jong RJ. Impact of severe malnutrition on short-term mortality and overall survival in head and neck cancer. Oral oncology. 2011; 47: 910-4

16. Isenring EA, Capra S, Bauer JD. Nutrition intervention is beneficial in oncology outpatients receiving radiotherapy to the gastrointestinal or head and neck area. British journal of cancer. 2004; 91 : 447-52.

17. Maccio A, Madeddu C, Gramignano G, Mulas C, Tanca L, Cherchi MC, et al. The role of inflammation, iron, and nutritional status in cancer-related anemia: results of a large, prospective, observational study. Haematologica. 2015; 100: 124-32.

18. Weiss G, Goodnough LT. Anemia of chronic disease. The New England journal of medicine. 2005; 352: 1011-23.

19. Becker A, Stadler P, Lavey RS, Hansgen G, Kuhnt T, Lautenschlager C, et al. Severe anemia is associated with poor tumor oxygenation in head and neck squamous cell carcinomas. International journal of radiation oncology, biology, physics. 2000; 46: 459-66.

20. Varlotto J, Stevenson MA. Anemia, tumor hypoxemia, and the cancer patient. International journal of radiation oncology, biology, physics. 2005; 63: 25-36. 
21. Vaupel P, Harrison L. Tumor hypoxia: causative factors, compensatory mechanisms, and cellular response. The oncologist. 2004; 9 Suppl 5: 4-9.

22. Vaupel $\mathrm{P}$, Thews $\mathrm{O}$, Hoeckel $\mathrm{M}$. Treatment resistance of solid tumors: role of hypoxia and anemia. Medical oncology. 2001; 18: 243-59.

23. Yasuda H. Solid tumor physiology and hypoxia-induced chemo/radio-resistance: novel strategy for cancer therapy: nitric oxide donor as a therapeutic enhancer. Nitric oxide : biology and chemistry. 2008; 19: 205-16.

24. Ong YK, Heng DM, Chung B, Leong SS, Wee J, Fong KW, et al. Design of a prognostic index score for metastatic nasopharyngeal carcinoma. European journal of cancer. 2003; 39: 1535-41.

25. Lin S, Tham IW, Pan J, Han L, Chen Q, Lu JJ. Combined high-dose radiation therapy and systemic chemotherapy improves survival in patients with newly diagnosed metastatic nasopharyngeal cancer. American journal of clinical oncology. 2012; 35: 474-9.

26. Hoskin PJ. Bisphosphonates and radiation therapy for palliation of metastatic bone disease. Cancer treatment reviews. 2003; 29: 321-7.

27. Yu HH, Tsai YY, Hoffe SE. Overview of diagnosis and management of metastatic disease to bone. Cancer control : journal of the Moffitt Cancer Center. 2012; 19: 84-91.

28. Chang J, Clark GM, Allred DC, Mohsin S, Chamness G, Elledge RM. Survival of patients with metastatic breast carcinoma: importance of prognostic markers of the primary tumor. Cancer. 2003; 97: 545-53.

29. Hung JJ, Jeng WJ, Hsu WH, Wu KJ, Chou TY, Hsieh CC, et al. Prognostic factors of postrecurrence survival in completely resected stage I non-small cell lung cancer with distant metastasis. Thorax. 2010; 65: 241-5.

30. Zhang S, Gao F, Luo J, Yang J. Prognostic factors in survival of colorectal cancer patients with synchronous liver metastasis. Colorectal disease : the official journal of the Association of Coloproctology of Great Britain and Ireland. 2010; 12: 754-61.

31. Tian YM, Zeng L, Wang FH, Liu S, Guan Y, Lu TX, et al. Prognostic factors in nasopharyngeal carcinoma with synchronous liver metastasis: a retrospective study for the management of treatment. Radiation oncology. 2013; 8: 272.

32. Cao X, Luo RZ, He LR, Li Y, Lin WQ, Chen YF, et al. Prognosticators and risk grouping in patients with lung metastasis from nasopharyngeal carcinoma: a more accurate and appropriate assessment of prognosis. Radiation oncology. 2011; 6: 104 